\title{
An Efficient Handoff Strategy for Mobile Computing Checkpoint System
}

\author{
Chaoguang Men ${ }^{1,2}$, Zhenpeng $\mathrm{Xu}^{2}$, and Dongsheng Wang ${ }^{1,2}$ \\ ${ }^{1}$ National Laboratory for Information Science and Technology, Tsinghua University, Beijing \\ 100084, China \\ ${ }^{2}$ Research Center of High Dependability Computing Technology, Harbin Engineering \\ University, Harbin, Heilongjiang, 150001, P.R. China \\ \{mencg, wds\} atsinghua.edu.cn, \\ \{menchaoguang, xuzhenpeng\} @hrbeu.edu.cn
}

\begin{abstract}
The Eager, Lazy and Movement-based strategies are used in mobile computing system when handoff. They result in performance loss while moving the whole checkpoint on fault-free or slow recovery while not moving any checkpoint until recovery. In the paper, a compromise strategy is proposed. The whole recovery information are broken into two parts, which one little part with high-priority should be transferred to the new cell during handoff and another large part with low-priority should be transferred only when the mobile host recovers from a fault. From the view of mobile host, it seems that all recovery information reside on the local mobile support station. The strategy guarantees little performance losing when fault-free and quick recovery when fault occurs. Experiments and analysis show the handoff strategy performance overcomes others.
\end{abstract}

Keywords: mobile computing, fault tolerant, checkpoint, handoff, rollback recovery.

\section{Introduction}

Checkpointing and rollback-recovery has been an attractive technique for providing fault-tolerance in mobile computing system [1]. Due to the mobility of the hosts, limited bandwidth, highly unreliable wireless link, mobile hosts disconnect from network voluntarily, power restriction and limitation of storage space in mobile devices, conventional checkpointing recovery schemes used in wired distributed network cannot be directly applied to mobile environment [2]. When a mobile host $(\mathrm{MH})$ moves from one cell to another, Eager, Lazy and Movement-based strategies are used, which move the whole recovery information to new mobile support station $(M S S)$ or not move any recovery information until a $M H$ recovers [3]. A strategy which moves the whole recovery information in fault-free will depress the performance of system due to transfer useless information and others which not move any recovery information until a fault occurs will delay the system recovers from a fault due to recovery information can not be gotten in time. No one strategy is 
excellent in every circumstance. A compromise strategy is proposed. Only a few part of recovery information is moved to the new local cell when a $\mathrm{MH}$ moves, which little useless work is done when fault-free or quick recovery can be done when a fault occurs.

The paper is organized as follows: Section 2 introduces the system model and definitions. Section 3 presents a checkpoint and recovery strategy with an efficient handoff scheme for mobile computing. Section 4 gives its correctness proofs. Section 5 compares the handoff scheme with others. Section 6 draws a conclusion.

\section{Preliminaries}

A mobile computing system $M C S=\langle N, C\rangle$ is composed of a set of nodes $N$ and a set of channels $C$. The set of nodes $N=M \cup S$ can be divided into two types, $M=\left\{M H_{1}\right.$, $\left.\mathrm{MH}_{2}, \ldots, \mathrm{MH}_{n}\right\}$ is the set of $\mathrm{MHs}$, which are able to move while retaining their network connections and $S=\left\{M S S_{1}, M S S_{2}, \ldots, M S S_{m}\right\}$ is the set of static nodes acting as the $M S S$ s. The set of channels $C=W \cup W^{\prime}$ can be divided into two disjoint sets, the set of high-speed wired channels $W$, where $W=S \times S$ is the type through that static nodes are connected, and the set of low bandwidth wireless channels $W^{\prime}$, where $W^{\prime}=S \times M$ is the type through that $M H$ s are connected to a MSS. A cell is a geographical area covered by a $M S S$. A $M H$ residing in the cell of $M S S_{\mathrm{p}}$ can directly communicate with $M S S_{\mathrm{p}}$ through a wireless channel. In a cell of $M S S_{\mathrm{i}}$, let $C L_{\mathrm{i}}=\left\{M H_{\mathrm{j}} \mid M H_{\mathrm{j}} \in M S S_{\mathrm{i}}, 0<j<n+1\right\}$ denotes the active nodes or sleeping nodes identified by Active_MH_List $t_{\mathrm{i}}$ or Disconnected_MH_List $t_{\mathrm{i}}$ respectively, then there exists a channel $\left\langle M S S_{\mathrm{i}}, M H_{\mathrm{j}}\right\rangle \in W^{\prime}$ only if $M H_{j} \in C L_{\mathrm{i}} \Rightarrow M H_{\mathrm{j}} \notin C L_{\mathrm{k}}, \forall k \neq i$, assuming that the geographical cells around each of the MSS do not overlap. The $M H$ s have limited battery power and hence cannot keep communication with the MSSs for long, hence they often disconnect from the network. Such disconnections can be voluntary without any fault or involuntary due to abruptly running out of battery. The mobile computing system model is described in Fig. 1.

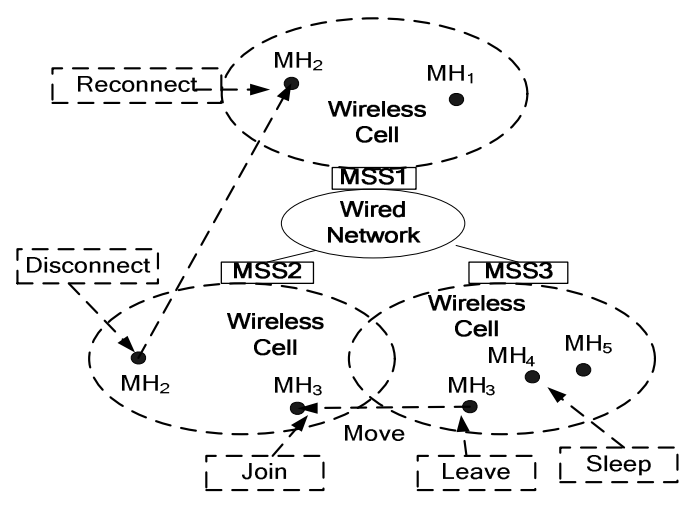

Fig. 1. Mobile computing system model 
Distributed computations running concurrently on different $\mathrm{MH}$ s consist of a set of $N$ processes denoted by $P_{1}, P_{2}, \ldots, P_{\mathrm{n}}$. Processes do not share a global memory or a global physical clock, and they communicate with each other only through message passing. For simplicity, we assume that only one process runs on each $M H$. So we can use the terms ' $M H$ ' and process interchangeably. We assume that each of the channels is bidirectional with reliable $F I F O$ delivery of messages and the message transfer delays are finite but arbitrary. Processes follow the piece-wise deterministic execution model, and the underlying computation is asynchronous. The fault model is assumed to be fail-stop and all faults can be detected immediately, which results in halting failed process, initiating recovery action are considered to be transient and the same fault would not repeat when the process restarts.

Let $R c v(i, \alpha)$ denotes the $\alpha$ th message receiving event of a process $P_{i}$; the state interval $I(i, \alpha)$ denotes the sequence of states generated between $\operatorname{Rcv}(i, \alpha-1)$ and $\operatorname{Rcv}(i, \alpha)$, where $\alpha>0$ and $R c v(i, 0)$ denotes the initial event. Then, the dependency relation of processes caused by the message communication can be defined as follows:

Definition 1. Dependency Relation: A state interval $I(i, \alpha)$ is said to be dependent on another state interval $I(j, \beta)$ if one of the following conditions is satisfied and the dependency relation is denoted by $I(j, \beta) \rightarrow I(i, \alpha)$ :

(i). $i=j$ and $\alpha=\beta+1$

(ii). For an event $R c v(i, \alpha)$, the corresponding message-sending event happens in $I(j, \beta)$

(iii). For any $I(k, \gamma), I(j, \beta) \rightarrow I(k, \gamma)$ and $I(k, \gamma) \rightarrow I(i, \alpha)[2]$.

With the pessimistic message logging scheme, an interval $I(i, \alpha+1)$ can be fully recovered after a fault if the event, $\operatorname{Rcv}(i, \alpha)$, has been stably logged; Otherwise, the interval becomes lost. During the rollback-recovery of a process, the dependency relation may cause an inconsistency problem.

Definition 2. Orphan interval: An interval on which depends any lost interval is called an orphan state interval.

Definition 3. Consistent Recovery: The recovery from a fault $F(i, f)$ is said to be consistent, if and only if there is not any orphan state interval, that is, for any $I(i, \alpha) \in L(i, f)$ there exists no $I(j, \beta)$, such that $I(i, \alpha) \rightarrow I(j, \beta)$. Where $F(i, f)$ denotes the $f$ th fault of $P_{i}$ and $L(i, f)$ denotes the set of lost state intervals caused by $F(i, f)$ [3].

The handoff and location scheme are supplied to support the mobility of $M H$. When a $\mathrm{MH}$ leaves a cell and enters another cell, it must end its current connection by sending a leave $(r)$ message to its local $M S S$, where $r$ is the sequence number of the last message received from the $M S S$. Then the $M H$ establishes a new connection by sending a join $(M H$-id, previous $M S S$-id) message to the new $M S S$. Usually, leaving a cell and entering another cell happens simultaneously when an $M H$ crosses the boundary between two cells and it is called a handoff. Each MSS maintains a list of identifiers of $M H \mathrm{~s}$ that are currently supported by the $M S S$. A $M H$ can also disconnect itself from the local MSS without leaving the cell by sending disconnect $(r)$ message when the $M H$ goes into the sleep mode for power conservation. Later, the $M H$ can reconnect to any $M S S$ by sending a reconnect $(M H$-id, previous $M S S$-id) message to 
the MSS. If the $M H$ is reconnected to a new MSS, the new MSS informs the previous $M S S$ of the reconnection of the $M H$ so that the previous $M S S$ can perform the proper handoff procedures [4].

Handoff time is an important parameter which affects mobile system performance besides checkpoint state-saving cost and recovery cost [5]. There three categories handoff strategy named Eager, Lazy and Movement-based. Eager mobility handoff strategy, which also named Pessimistic, always keeps the logging and checkpoint information in the local $M S S$ in which the $M H$ currently resides [5]. Thus, when the $M H$ moves from one $M S S$ to another during the execution of a mobile application, all the checkpoint and logging information must be moved to the current MSS as well. The advantage of this approach is fast failure recovery. But the MSSs visited by the $M H$ have to experience high fault-free cost to transfer the recovery information and access the stable storage. Under the Lazy strategy, on the other hand, the checkpoint and logging information do not be moved as the $M H$ moves [5]. Rather, a forwarding pointer is established from the local MSS to the last MSS so that when a failure occurs, the checkpoint and logging information of the mobile application can be recovered from all the MSSs on the forwarding chain by following the links. The advantage of this approach is little fault-free cost, but the recovery cost can be too high, if the recovery information is dispersed over a wide range of cells. The tradeoff schemes are Movement-based handoff strategies, which are Distance-based and Frequency-based [4]. Under the Distance-based scheme, which focuses on the distance between $M H_{i}$ and the $M S S$ carrying latest checkpoint of $M H_{i}$, the checkpoint and message logs need to be moved into a $M S S$ near $M H_{i}$, only when the moving distance of $M H_{i}$ from a $M S S$ carrying the latest checkpoint exceeds a certain threshold. On the other hand, the Frequency-based scheme concerns the number of handoffs, since that number indicates the number of sites carrying the message logs and the frequency of communication for collecting the message logs in case of recovery. Hence, in this scheme, $M H_{i}$ keeps counting the number of handoff and transfers the checkpoint and logs if the number exceeds a certain value. Of course, in both of the above schemes, the recovery cost and the fault-free operation cost is adjustable using the threshold values. Checkpoint and logs are moved to new local MSS when fault-free, the Movement-based schemes have the disadvantage of Eager. Checkpoint and logs are not moved to new local MSS until recovery, the Movement-based schemes have the disadvantage of Lazy. Obviously, how effective these strategies would be depends on various system parameters, including the checkpoint rate, logging message arrival rate, user mobility rate, failure rate, and bandwidth. No one scheme is always better than others under all situations [6].

\section{The Recovery Scheme}

The proposed recovery scheme is based on independent checkpointing, pessimistic message logging and asynchronous rollback-recovery. An efficient handoff scheme is proposed. Different from Eager, Lazy and Movement-based schemes which move the whole recovery information or do not move any recovery information until recovery, in our strategy, the whole recovery information which include checkpoint and logs are broken into two parts. One part includes only a little part of recovery information with 
high-priority. The other part includes the rest of the recovery information with lowpriority. The high-priority part of recovery information is treated as that in Eager scheme and the low-priority part of recovery information is treated as that in Lazy scheme. Appropriate partitions high-priority and low-priority recovery information can satisfy both quick recovery and fault-free cost. When recovering from a fault, the high-priority part can be transferred instantly to the recovering $M H$, the low-priority part can be collected by the local MSS simultaneously from other MSSs and then be transferred to the recovering $M H$ successively. From the view of the recovering $M H$, it seems that all recovery information always resides on the local MSS.

\subsection{The Data Structure and Denotations}

Let $C K_{i, \alpha}$ denotes the $\alpha$ th checkpoint of $M H_{\mathrm{i}} ; C K_{-} i n f o_{\mathrm{i}}$ is a record which contains six variables, $C K \_s n, C K \_l o c, C K \_l o w, L o g m \_s e q$, Send_max, and Log_queue. CK_sn denotes the sequence number of the latest checkpoint and the $C K \_l o c$ denotes the identifier of the MSS carrying the high-priority of the latest checkpoint; $C K_{-}$low denotes the identifier of MSS carrying the low-priority of the latest checkpoint; Logm_seq denotes the sequence number of the first message logged after the latest checkpoint; Send_max denotes the maximum sequence number of message sent successfully by $M H_{\mathrm{i}}$ to other $\mathrm{MHs}$ since the latest checkpoint; Log_queue is a list established for the local MSS to save the identifiers of MSSs which have the logs saved after the latest checkpoint; $M s g_{i, \alpha}$ denotes the $\alpha$ th message sent by $M H_{\mathrm{i}}$; Rcv_seq is an integer variable, which denotes the maximum sequence number of messages that have been received and consumed in $M H_{\mathrm{i}} . \operatorname{Logm}_{i, \alpha}$ denotes the $\alpha$ th message log.

\subsection{The Checkpointing and Logging}

Each $M H_{\mathrm{i}}$ takes an initial checkpoint on initialization and sets the corresponding checkpoint sequence number $C K_{-}$info $o_{\mathrm{i}} . \mathrm{CK}_{-} s n$ to 0 . Every $M H$ takes checkpoint periodically. When $M H_{\mathrm{i}}$ finishes a new checkpoint, the information about this checkpoint is recorded in $C K \_i n f o_{\mathrm{i}}$. The $C K \_i n f o_{\mathrm{i}}$ and the new checkpoint will be sent to its local $M S S_{\mathrm{p}}$.

Each MSS logs the received messages before delivering to $M H \mathrm{~s}$ in its cell. As a message heading for $M H_{\mathrm{i}}$ should be routed through the local $M S S_{\mathrm{p}}$, using the local $M S S_{\mathrm{p}}$ to $\log$ the message into its storage space will not incur extra overhead. $M S S_{\mathrm{p}}$ also logs the messages of the mobility of $\mathrm{MHs}$, including the messages of $\mathrm{MHs}$ to join in, leave from, disconnect from and reconnect to the cell. Upon a user input of the $M H$, a copy of it is firstly forwarded to the local $M S S_{\mathrm{p}}$ for logging in case of its lost. On receipt of the acknowledgment from $M S S_{\mathrm{p}}$, the $M H$ starts to process the input event. When $M H_{\mathrm{i}}$ leaves or disconnects from $M S S_{\mathrm{q}}$, it sends Disconnect $(i)$ message to the local $M S S_{\mathrm{q}}$ for logging. $M S S_{\mathrm{q}}$ logs the event on the receipt of it and deals with it. When $M H_{\mathrm{i}}$ joins in a new cell of MSS, says $M S S_{\mathrm{r}}$, it sends $\operatorname{Join}\left(M S S_{\mathrm{q}}\right)$ to $M S S_{\mathrm{r}}$. And $M S S_{\mathrm{r}}$ will add $M S S_{\mathrm{p}}$ into the $C K_{-}$info $_{\mathrm{i}} \cdot \log _{-} q u e u e$ if $M S S_{\mathrm{p}}$ is not in the CK_info. Log_queue. $_{\text {. }}$

The checkpointing and message logging algorithm is described in Fig. 2. 


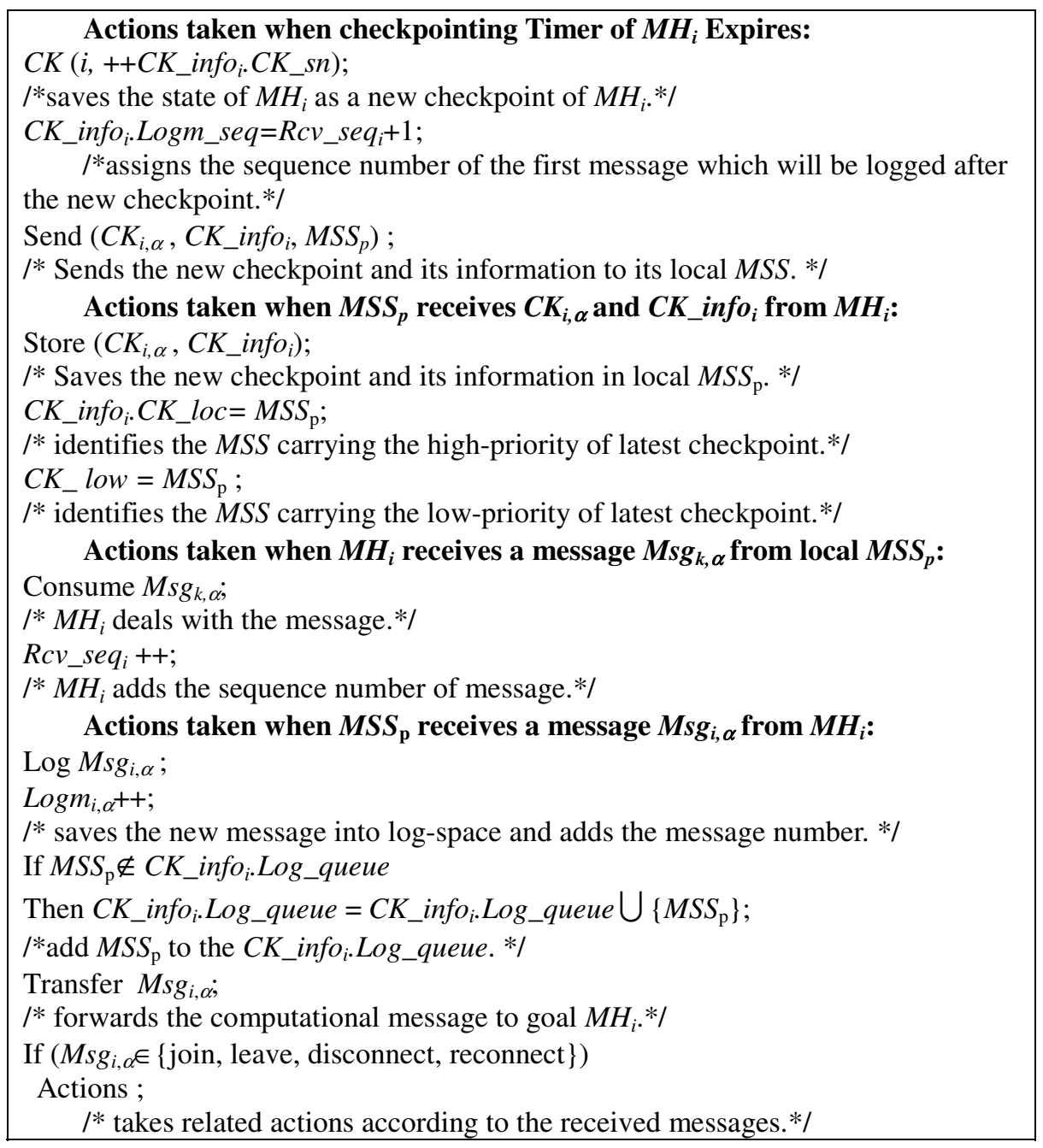

Fig. 2. Checkpointing and message logging algorithm

\subsection{The Handoff Strategy}

The recovery information including checkpoint and massage logs are broken into two parts that one with high-priority and the other with low-priority. The main idea is that low-priority checkpoint information can be sent to the local MSS of recovering $M H$ through high speed wired network at the same time as the high-priority recovery information is being sent to recovering $M H$ through low speed wireless network.

The amount of high-priority part and low-priority part of recovery information depend on the speeds of wired and wireless networks. Set $L T_{\max }$ and $W T_{\min }$ denote the maximum communication speed of wireless network and minimum communication speed of wired network respectively. Set $V P_{0}$ denotes the amount of high-priority 
recovery information. For simplicity, we assume that the amount transmitted is the integral multiple of packet size.

When a $M H_{i}$ recovers from a fault, the $V P_{0}$ will be sent to $M H_{i}$ from the local $M S S_{p}$ instantly. The transmission time at least is:

$$
t_{1}=\frac{V P_{0}}{L T_{\max }} .
$$

The amount of low-priority recovery information collected from other MSSs simultaneously in time $t_{1}$ at least is:

$$
V P_{1}=t_{1} * W T_{\min }=\frac{V P_{0} \times W T_{\min }}{L T_{\max }}
$$

Because the speed of wired network is faster than the speed of wireless, so $V P_{1}>V P_{0}$, that is more information can be collected through wired network when an amount of information is sent through wireless network. In turn, more recovery information, $V P_{2}$, can be collected through wired network when $V P_{1}$ is sent through the wireless network. The amount of recovery information transferred to $M S S_{p}$ from other MSSs is:

$$
V P_{0} \times\left[\frac{W T_{\min }}{L T_{\max }}+\left(\frac{W T_{\min }}{L T_{\max }}\right)^{2}+\cdots+\left(\frac{W T_{\min }}{L T_{\max }}\right)^{n-1}+\left(\frac{W T_{\min }}{L T_{\max }}\right)^{n}\right] .
$$

The effective handoff scheme is described in Fig. 3.

\section{Actions taken when $M S S_{p}$ receives join $\left(M S S_{q}\right)$ request:}

Send $\left(V P_{0 \_}\right.$retrieve, $\left.M S S_{q}\right)$

/*sends request message to $\boldsymbol{M S S}_{q}$ get high-priority part of $C K_{i, \alpha} * /$

Actions taken when $M S S_{q}$ receives $V_{P_{0}}$ retrieve from the new local $M S S_{p}$ :

Send $\left(V P_{0}, M S S_{\mathrm{p}}\right)$

$/ *$ sends high-priority part of recovery information to $M S S_{\mathrm{p}}$ * */

Actions taken when $M S S_{\mathrm{p}}$ receives $V P_{0}$ :

$C K \_l o c=M S S_{\mathrm{p}}$;

Store $C K_{i}$ info $o_{i}$ and high-priority part of $C K_{i, \alpha}$;

$/ *$ identifies the $M S S$ carrying the high-priority of latest checkpoint and saves the high-priority of recovery information.*/

Fig. 3. An effective handoff scheme

\subsection{Independent Recovery}

When recovering from a fault, $M H_{i}$ sends a recovery request, $\operatorname{RollbackReq}(i)$, to its local $M S S_{p}$. If $C K_{-}$info $o_{i}$ is saved in the local $M S S_{p}$, the high-priority part of recovery information is sent to $M H_{i}$ instantly. To collect the rest of checkpoint and logs, the

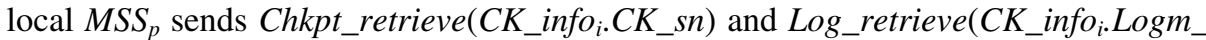
seq) request to other $M S S$ s according to $C K \_l o w$ and $C K \_$info $o_{i}$ Log_queue. After receiving the request, the other $M S S$ s reply with the low-priority checkpoint part and 
the logs whose sequence number is not less than $C K \_i n f o_{i}$.Logm_seq. After transferrring the high-priority part of recovery information to the recovering $M H_{i}$, the local $M S S_{p}$ sends the low-priority checkpoint part to the recovering $M H_{i}$ successively. After receiving the whole checkpoint, $M H_{i}$ reloads the checkpoint to restore the system, and then resumes and replays the logs. During recovering, new message sent to $M H_{i}$ is saved in its local $M S S_{p}$, and will be forwarded to $M H_{i}$, in turn, after recovering. The message that sequence number less than $C K \_i n f o_{i}$.Logm_seq is discarded to avoid repeat messages.

If there is not $C K_{-}$info $o_{i}$ in the local $M S S_{p}$, it means that a fault has occurred in other cell and then $M H_{i}$ enters this cell in where it submits the recovery request. $M S S_{p}$ broadcasts recovering request to all $M S S$ s. The previous local $M S S_{q}$ sends the highpriority part of recovery information to $M S S_{p}$. $M S S_{p}$ executes the recovering process. The asynchronous recovery algorithm is described in Fig. 4.

\section{Actions taken when $\mathrm{MH}_{\boldsymbol{i}}$ occurs a fault:}

Send $\left(\right.$ RollbackReq $\left.(i), M S S_{p}\right)$;

/*Sends recovery request to the local $M S S_{p} *$ /

Actions taken when $M_{S S}$ receives RollbackReq(i) from $\boldsymbol{M H}_{i}$ :

If $\left(M H_{i} \in\left(\right.\right.$ Active_MH_Lis $t_{p}$ or Disconnected_MH_List $\left.\left.t_{p}\right)\right)$

Send $\left(V P_{0}, M H_{i}\right)$;

/*if $M S S_{p}$ holds latest checkpoint with high-priority, sends it to $M H_{i .}$ */

Send (Chkpt_retrieve (CK_info ${ }_{i}$. $\left.\left.C K \_s n\right), C K \_l o w\right)$;

$/ *$ sends retrieval message to $C K \_l o w$ to get the remnant part of checkpoint.*/

Send (Log_retrieve $\left(C K \_\right.$info ${ }_{i}$.Logm_seq), CK_info ${ }_{i}$.Log_queue);

$/ *$ sends request to $M S S_{r}$ in the list of Cpinfo.Log_queue to get message logs.*/

Else

Broadcast info_retrieve $(i)$;

${ }^{*}$ If the local $M S S_{p}$ don't hold the latest $C K \_i n f o_{i}$, broadcasts the recovery request. $* /$

Actions taken when $M S S_{k}$ receives Broadcast info_retrieve(i) from $M S S_{p}$ :

Send (high-priority of $C K_{i, \alpha}, C K_{-}$info $o_{i}, M S S_{p}$ );

$/ *$ sends the high-priority part of recovery information to local $M S S_{p}$.*/

Actions taken when $M S S_{q}$ receives Chkpt_retrieve $\left(C K \_s n\right)$ from $M S S_{p}$ :

Send (the remnant of $C K_{i, \alpha}, M S S_{p}$ );

$/ *$ sends the remnant of checkpoint to local $M S S_{p} * /$

Actions taken when $M S S_{q}$ receives $L o g \_$retrieve $\left(L o g m \_s e q\right)$ from $M S S_{p}$ :

If $\left(\exists \operatorname{Logm}_{i, \alpha}\right.$ in $M S S_{q} \log$ space and $\alpha \geq L o g m_{-}$seq $)$

Send $\left(\operatorname{Logm}_{i, \alpha}, M S S_{p}\right)$;

$/ *$ sends related message logs to local $M_{S S}$ * */

Actions taken when $\mathrm{MH}_{i}$ receives the full $\mathrm{CK}_{i, \alpha}$ from $M S S_{p}$ :

Restore $C K_{i, \alpha}$;

/*restores the full checkpoint.*/

Resume action;

/*starts the computation process of recovery.*/

Fig. 4. The asynchronous recovery algorithm 


\subsection{Garbage Collection}

$M H_{\mathrm{i}}$ takes new checkpoint $C K_{i, \alpha}$ and sends the checkpoint and $C K_{-}$info $o_{i}$ to $M S S_{p}$. $M S S_{p}$ sends a message which a new checkpoint has been taken to all MSSs which will delete old checkpoint $C K_{i, \alpha-1}$ and relative information that denoted by old $C K \_$info ${ }_{i}$. $C K \_l o c, C K \_$info ${ }_{i}$. $C K \_l o w, C K \_$info ${ }_{i}$. Log_queue. Every $M S S$ will release the space held by checkpoint $C K_{i, \alpha-1}$ and implement garbage collection.

\section{Correctness of the Algorithm}

Theorem 1 . The asynchronous recovery of $M H_{i}$ is a consistent recovery.

Proof: Recoverable: the latest checkpoint and the messages with the sequence number larger than the $C K_{-}$info . $_{i} \operatorname{logm} \_s e q$ were saved safely due to the reliable communication, the reliable $M S S$ s and the pessimistic message logging. Therefore, on the recovery of $M H_{i}$, every message logs and the latest checkpoint can be retrieved. The messages can be replayed according to the sequence number after restoring the latest checkpoint. In other words, $M H_{i}$ can reconstruct one possible sequence of state intervals as those constructed before the fault due to processes following the piecewise deterministic execution model. So $M H_{i}$ is recoverable on fault in the strategy.

Consistent recovery: The lost events which incurs $L(i, f)$ can only be the messages or user inputs that had not been sent successfully to the local MSS before a fault. This implies the corresponding messages could not be transferred to their destinations. According to the definition 1, the lost events can not incur new dependency relations between $M H$ s. Therefore, for any $I(i, \alpha) \in L(i, f)$ there exists no $I(j, \beta)$, such that $I(i, \alpha) \rightarrow$ $I(j, \beta)$. The independent recovery is a consistent recovery as the recovery strategy satisfies the definition 3 .

\section{Performance Study}

The model and parameters in [6] are adopted. MHs communicate with MSSs through 802.11a wireless network adapter. $M H$ moves from one cell to another follows a Poisson process with rate $\sigma=0.01$. The message sending rate of a $M H$ follows a Poisson process with rate $\lambda=0.1$. Each $M H$ takes a checkpoint with a fixed interval $T c=1000$ s, the failure rate of each $M H$ follows an exponential distribution with rate $\delta=0.0001$. Increment strategy is adopted for saving a checkpoint and its size is $1 \mathrm{MB}$. The size of a logs entry is $50 \mathrm{~B}$. The ratio of wireless network speed to wired network speed is $r=0.1$. The time required to load a log entry through a wireless channel is $T_{1}=0.016 \mathrm{~s}$, and the time required to load a checkpoint through a wireless channel is $T_{3}=0.32 \mathrm{~s}$. The time required to execute a $\log$ entry is $T_{2}=0.0008 \mathrm{~s}$. We assume that when a $M H$ moves 5 times, its checkpoint should be moved to the new local MSS in Frequency-based strategy and when a $M H$ moves 10 times, its checkpoint should be moved to the new local MSS in Distance-based strategy.

Fig. 5 shows the amounts of recovery information needed to be transmitted in every recovery strategies. The $y$-axis indicates the overhead of message transfer incurred by different strategies for $M H$ 's recovery, while the $x$-axis denotes the time that a fault 
occurs on the $M H$. The overhead of recovery information management under our virtual strategy is always less than those under Eager, Frequency-based and Distancebased strategy, and only a little large than that under Lazy strategy, because our compromise strategy only moves little, not the whole, latest checkpoint to the local $M S S$ when a $M H$ moves from one cell to another.

Fig. 6 shows the amounts of recovery information needed be transmitted by every strategy under different message sending rate after the system has run 500 seconds. The overhead of every handoff strategy becomes increment with the increment of message sending rate $\lambda$. The overhead of our handoff strategy almost equals to that under Lazy and far less than the other's.

Let $N(t)$ denotes the number of logs saved until $M H$ faults. $f_{f}(t)$ denotes the probability when a fault occurs in time $t$. Under our and Eager strategies, the recovering probability of time $T$ is [6]:

$$
F_{1}(T)=\sum_{M=0}^{+\infty} \int_{M T c}^{(M+1) T c} \operatorname{Pr}\left\{N(t)\left[T_{1}+T_{2}\right]+T_{3} \leq T\right\} f_{f}(t) d t .
$$

$M$ denotes the checkpoint number experienced by a $M H$ in time $t, T_{1}, T_{2}$ and $T_{3}$ denote the mean time of loading a log entry through wireless network, executing a log entry and loading whole checkpoint through wireless network respectively. $N(t)$ is a Poisson process with rate $\lambda=0.1$, and $f_{f}(t)$ is an exponential distribution with rate $\delta=0.0001$, we get:

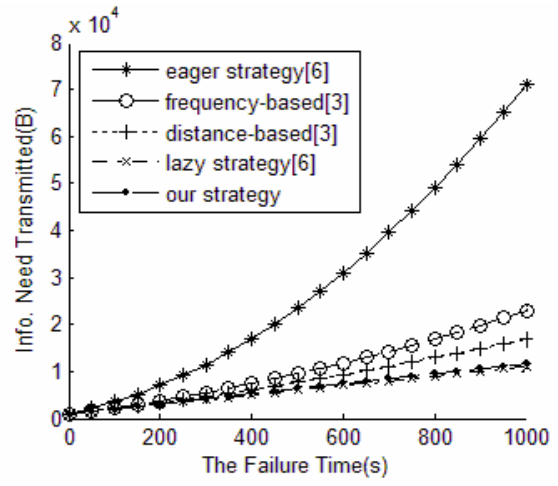

Fig. 5. The amounts of recovery information need transmitted

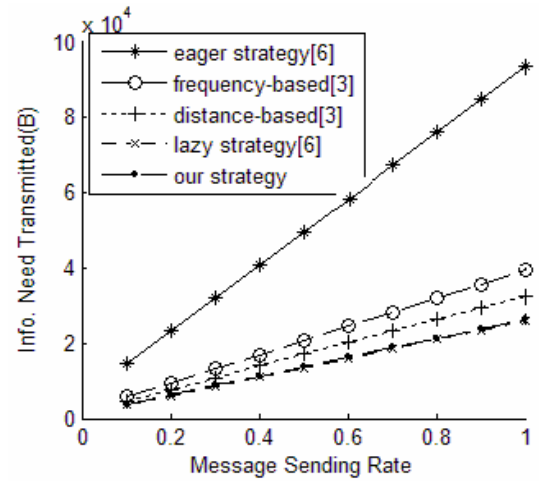

Fig. 6. The amounts of recovery information need transmitted with different rate

$$
\begin{aligned}
F_{1}(T) & =\sum_{M=0}^{+\infty} \int_{0}^{T c} \sum_{n=0}^{\left.\frac{T-T_{3}}{T_{1}+T_{2}}\right\rfloor} \frac{e^{-\lambda t^{\prime}}\left(\lambda t^{\prime}\right)^{n}}{n !} \bullet \delta e^{-\delta t^{\prime}} \bullet e^{-\delta M T c} d t^{\prime} \\
& =\frac{\int_{0}^{T c} \sum_{n=0}^{\left.\frac{T-T_{3}}{T_{1}+T_{2}}\right\rfloor} \frac{e^{-\lambda t}(\lambda t)^{n}}{n !} \bullet \delta e^{-\delta t} d t}{1-e^{-\delta T c}} .
\end{aligned}
$$


Under Lazy strategy, the recovering probability of time $T$ is:

$$
\begin{aligned}
F_{2}(T) & =\sum_{M=0}^{+\infty} \int_{M T c}^{(M+1) T c} \operatorname{Pr}\left\{T_{r} \leq T \mid t_{f}=t, k_{b}=k\right\} \\
& \bullet \operatorname{Pr}\left\{k_{b}=k \mid t_{f}=t\right\} f_{f}(t) d t .
\end{aligned}
$$

Replacement $N(t)$ and $f_{f}(t)$ by their value:

$$
\begin{aligned}
F_{2}(T) & =\sum_{M=0}^{+\infty} \int_{0}^{T c} \sum_{k=0}^{+\infty} \sum_{n=0}^{\left\lfloor\frac{T-T_{3}-r T_{3}-r k T_{3}}{r T_{1}+T_{1}+T_{2}}\right\rfloor} \frac{e^{-\lambda t^{\prime}}\left(\lambda t^{\prime}\right)^{n}}{n !} \bullet \frac{e^{-\sigma t^{\prime}\left(\sigma^{\prime}\right)^{k}}}{k !} \bullet \delta e^{-\delta t^{\prime}} \bullet e^{-\delta M T c} d t^{\prime} \\
& =\frac{\int_{0}^{T_{c}} \sum_{k=0}^{+\infty} \sum_{n=0}^{\left\lfloor\frac{T-T_{3}-r T_{3}-r k T_{3}}{r T_{1}+T_{1}+T_{2}}\right\rfloor} \frac{e^{-\lambda t}(\lambda t)^{n}}{n !} \bullet \frac{e^{-\sigma t(\sigma t)^{k}}}{k !} \bullet \delta e^{-\delta t} d t}{1-e^{-\delta T c}} .
\end{aligned}
$$

Fig. 7 shows the probabilities of recovering time under various handoff strategies. Our strategy which only has a little overhead large than that under Eager is better than that under Lazy and has the same recovery probability as Eager has. Fig. 8 shows the executing overhead under our asynchronous recovery strategy and coordinated recovery strategy which the number of $\mathrm{MHs}$ is 100 and only $10 \mathrm{MHs}$ need recovery from a fault. As shown in the figure, our strategy is better than coordinated strategy, and is more effective.

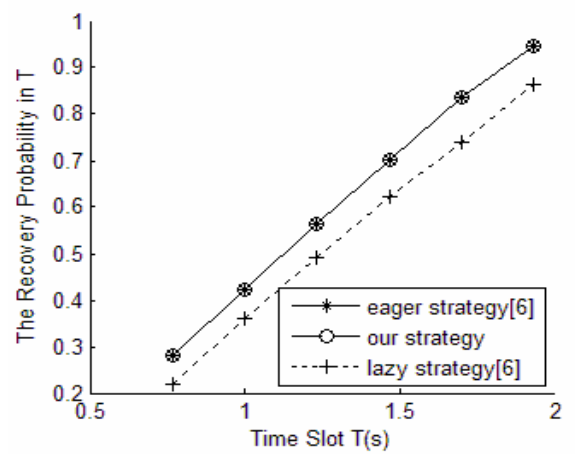

Fig. 7. The fault recovery probability

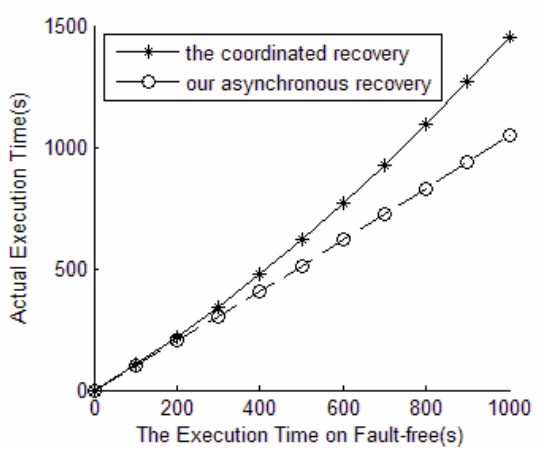

Fig. 8. The actual execution time of the mobile applications

\section{Conclusion}

The handoff strategy taken when a $M H$ moves from one cell to another will affect the executing efficiency and recovering time of checkpoint algorithm. Different from other schemes, in the strategy proposed in the paper, the recovery information is 
broken into two parts, which the first part must be transferred instantly to the new cell when a handoff happens and the second part can be transferred simultaneously to the local MSS through static network as the first part is transferred to the recovering $M H$. The partition principle is that the first part as little as possible and the second part as large as possible under guaranteeing recovering information to be transmitted to recovering $M H$ successively. From the view of recovering $M H$, it seems that all recovery information resides on the local $M S S$ all the time. This strategy considers both minimum executing time on fault-free and quickly recovering from a fault. Experiments and analysis show our strategy is better than others.

\section{References}

1. Elnozahy, E.N., Alvisi, L., Wang, Y.M., Johnson, D.B.: A Survey of Rollback-Recovery Protocols in Message-Passing Systems. ACM Computing Surveys 34(3), 375-408 (2002)

2. Ching, E.Y., Phipatanasuphorn, V.: A Survey of Checkpoint-Recovery Techniques in Wireless Networks (2002), http://www.cae.wisc.edu/ ece753/papers/Paper_9.pdf

3. Park, T., Woo, N., Yeom, H.Y.: An Efficient Optimistic Message Logging Scheme for Recoverable Mobile Computing Systems. IEEE Transactions on Mobile Computing 1(4), 265-277 (2002)

4. Park, T., Woo, N., Yeom, H.Y.: An Efficient Recovery Scheme for Mobile Computing Environments. In: The 8th International Conference on Parallel and Distributed Systems (ICPADS), Kyongju City, Korea, pp. 53-60 (2001)

5. Pradhan, D.K., Krishna, P., Vaiday, N.H.: Recoverable Mobile Environment: Design and Trade-off Analysis. In: Proc. of the 26th Int'l Symp. on Fault Tolerant Computing System, Sendai, Japan, pp. 16-25 (1996)

6. Chen, I.-R., Gu, B., George, S.E., Cheng, S.-T.: On failure recoverability of client-server applications in mobile wireless environments. IEEE Transactions on Reliability 54(1), $115-122$ (2005) 